Published in final edited form as:

Complement Ther Med. 2017 February ; 30: 40-53. doi:10.1016/j.ctim.2016.11.007.

\title{
Sanfu acupoint herbal patching for stable asthma: A systematic review and meta-analysis of randomised controlled trials
}

\author{
Fen Zhou ${ }^{a, b}$, Ning Liang ${ }^{b}$, Manfred Maier ${ }^{c}$, and Jian-ping Liu ${ }^{b, d,{ }^{*}}$ \\ aSchool of Nursing, Beijing University of Chinese Medicine, Beijing, 11 Bei San Huan Dong Lu, \\ Chaoyang District, Beijing, 100029, China \\ ${ }^{b}$ Center for Evidence-Based Chinese Medicine, Beijing University of Chinese Medicine; 11 Bei \\ San Huan Dong Lu, Chaoyang District, Beijing, 100029, China \\ 'Department of General Practice, Center for Public Health, Medical University of Vienna, \\ Kinderspitalgasse 15/1. Stock, A-1090, Vienna, Austria \\ dNational Research Centre in Complementary and Alternative Medicine (NAFKAM), Faculty of \\ Health Sciences, UiT The Arctic University of Norway, Tromsø, Norway
}

\begin{abstract}
Background-Sanfu acupoint herbal patching (SAHP) is extensively used in people with stable asthma in China. However, the evidence available is scarce. This systematic review aims to evaluate the preventive and therapeutic effect and safety of SAHP in people with stable asthma.

Methods-We searched seven electronic databases for randomised controlled trials (RCTs). The Cochrane risk of bias tool was utilised to evaluate the methodological quality of the included studies and RevMan 5.3 and GRADEpro 3.6.1 were applied to perform data analyses.
\end{abstract}

Results-A total of 34 RCTs involving 3313 participants were included. The overall methodological quality of the trials was of high risk of bias. SAHP plus conventional therapy (CT) decreased the mean frequency (times per year) of asthma exacerbations compared with $\mathrm{CT}$ alone (MD: $-1.42 ; 95 \%$ CI: -2.19 to $-0.65 ; 7$ RCTs), and similar effect was found for SAHP versus sham SAHP (MD: 0.42; 95\%CI: 0.26-0.69; 1 RCT). For lung function (including PEF\%, $\mathrm{FEV}_{1} \%$ and $\mathrm{FEV}_{1} / \mathrm{FVC}$ ), SAHP plus $\mathrm{CT}$ showed better effect than $\mathrm{CT}$ alone, and so did SAHP versus sham SAHP on PEF and PEF\%. Adverse effects in the SAHP groups were reported to be mild and well tolerated.

Conclusions-SAHP alone or combined with CT appears to be more effective than sham SAHP or CT on reduction of asthma exacerbations, improving lung function, and SAHP seems to be safe.

*Corresponding author at: Center for Evidence Based Chinese Medicine, Beijing University of Chinese Medicine, 11 Bei San Huan Dong Lu, Chaoyang District, Beijing, 100029, China, jianping_1@ hotmail.com, liujp@bucm.edu.cn.

Author contributions

Conceived and designed the review: F Zhou and JP Liu; Searched studies: F Zhou and N Liang; Appraised trials: F Zhou, N Liang and JP Liu; Extracted data: F Zhou and N Liang; Synthesized and analyzed date: F Zhou and JP Liu; Wrote the paper: F Zhou, JP Liu and M Maier.

Conflict of Interests

None declared. 
However, the findings should be interpreted with caution due to limitations in trial quality. Further, rigorously designed, large-scale trials are warranted for robust evidence.

\section{Keywords}

Stable asthma; Acupoint herbal patching; Sanfu; Traditional Chinese medicine; Systematic review; Randomised controlled trials

\section{Introduction}

Asthma is defined as a chronic airway inflammation with variable expiratory airflow limitation and a history of respiratory symptoms such as wheeze, shortness of breath, chest tightness and cough that vary over time and in intensity. ${ }^{1}$ The prevalence of asthma in adults was estimated to be $4.3 \%$, ranging from $0.2 \%$ in China to $21.0 \%$ in Australia. ${ }^{2}$ It's still increasing in many parts of the world from 300 millions up to 400 million by $2025 .{ }^{3}$ The burden of asthma is similar to that of diabetes or Alzheimer disease when calculating disability-adjusted life years. ${ }^{4}$ Moreover, when the asthma symptoms are uncontrolled, or one or more severe exacerbation happened in last 12 months, this could increase the risk of recurrence of the next exacerbation. ${ }^{5,6}$ This vicious circle might increase the risk of asthma related death. ${ }^{7}$ Therefore, treatment and management of stable asthma to prevent acute exacerbation are critical.

Currently, conventional therapy (CT) for stable asthma includes pharmacological and nonpharmacological interventions. Pharmacological medications mainly include corticosteroids, beta $_{2}$-agonists, leukotriene receptor antagonist and sustained-release theophylline for control and relief of asthmatic symptoms. However, inappropriate use of inhaler technique ${ }^{8}$ and poor adherence ${ }^{9}$ are the major problems. On the other hand, besides the avoidance of exposure to allergens and air pollution, non-pharmacological interventions are often used including allergen immunotherapy, bronchial thermoplasty and vaccines. However, it should take into account the risk of adverse effects, the inconvenience and cost of the prolonged course of the therapy. ${ }^{1}$

In China, the use of acupoint herbal patching (AHP, also called as acupuncture point application therapy) in asthma management was first recorded in Zhang Shi Yi Tong in Qing Dynasty (1644-1912), ${ }^{10}$ and is still in popular use nowadays. As a traditional Chinese medicine (TCM) technique, Sanfu AHP (SAHP) is the external application of processed medicinal herbal preparations directly to specific acupuncture points (acupoints), only during the sanfu period to produce preventive and/or therapeutic effects. According to the Lunar calendar, fu refers to the hottest period of the year between mid-July to mid-August, lasting 30 to 40 days. Each ten days is called one fu, and three fus are called as sanfu. SAHP is a comprehensive intervention that comprises percutaneous absorption of applied herbal extracts, stimulation and regulation of meridians and acupoints.

According to TCM theory, usually, asthma is attributed to lung obstructed by long-retained phlegm. ${ }^{11}$ When exogenous pathogenic, improper diet, emotional disturbance, over strain attack patient could make asthma recurrence. ${ }^{11} \mathrm{TCM}$ also believe lung, spleen and kidney are related to immunology functions; while AHP might regulate these three organs (Zangfu) 
through herbs absorption and meridians stimulation. ${ }^{12,13}$ AHP contains herbs with acridwarm and penetrating flavor which might warm the lung, remove phlegm, facilitate $q i$ flow, dissolve masses, dredge the collaterals. Additionally, because sanfu is of special significance in TCM when yang (a moral connotation of light) ${ }^{14}$ in human body is the strongest, ${ }^{12}$ it is considered as most effective when AHP is applied during sanfu period. A study found that SAHP could reduce the IL-4, and increase IFN- $\gamma$ significantly which might be the one of the mechanisms of asthma management. ${ }^{15}$ A systematic review of six trials published in 2015 showed a positive effect of AHP only on immune outcomes for children with asthma. ${ }^{16}$ Another review of 16 RCTs published recently with insufficient searches and mainly focusing on pulmonary function showed encouraging but inconclusive effect. ${ }^{17}$

For this review, we included much larger number of trials (34 RCTs), and evaluated the preventive and therapeutic effect and safety of SAHP alone or in combination with CT for stable asthma, using patient related outcomes such as asthma exacerbations.

\section{Methods}

This review was prepared according to the Preferred Reporting Items for Systematic Reviews and Meta-Analyses (PRISMA) guidelines. ${ }^{18}$

\subsection{Protocol and registration}

The protocol registration number was CRD42015019337 (http://www.crd.york.ac.uk/ Prospero).

\subsection{Eligibility criteria}

We included randomised controlled trials (RCTs) (parallelgroup, cross-over or cluster) using SAHP in stable asthma regardless of blinding, publication type or language. Quasirandomised trials were excluded. Participants included both children and adults with stable asthma (not during exacerbation) regardless of gender, etiology, ethnic, and course of the disease. Interventions included all types of SAHP alone or in combination with conventional therapy (CT) and/or biological agents. CT means that prevention and treatment of stable asthma is based on the Global Strategy for Asthma Management and Prevention (GINA) guidelines ${ }^{1}$ or its predecessor documents. No restrictions were applied to the herbal regimen used, acupoints selected, number of SAHP sessions or treatment course. The controls included no treatment, sham SAHP, or CT alone. In addition, co-intervention(s) were also included as long as they were applied in both arms.

RCTs had to assess at least one of the following outcomes: frequency of asthma exacerbations during follow-up (primary outcome); asthmatic symptoms by validated instruments (including symptom scores, Likert scale, visual analogue scale); lung function (peak expiratory flow (PEF), predicted peak expiratory flow (PEF\%), predicted forced expiratory volume in one second $\left(\mathrm{FEV}_{1} \%\right), \mathrm{FEV}_{1} / \mathrm{FVC}$ (forced vital capacity)) by spirometry; quality of life measured by a validated asthma-specific or generic instruments; numbers of participants experiencing each adverse events. 


\subsection{Search methods}

We searched for published studies in seven electronic databases from their inception to June 2015: PubMed, the Cochrane Central Register of Controlled Trials, EMBASE, China National Knowledge Infrastructure (http://oversea.cnki.net/kns55/default.aspx), Chinese Biomedical Literature Database (www.sinomed.ac.cn), Chinese Science and Technology Periodical Database (http://en.cqvip.com), and Wan fang Database (www.wanfangdata.com). In order to identify unpublished studies, we searched five major trial registries (Supplementary file. S1).

Moreover, we also hand-searched the reference lists of all retrieved papers for additional relevant reports. A filter was applied to limit to humans. Details of the search strategies are available from Supplementary file 1.

\subsection{Study selection and data collection process}

Extracted data included participants (age, gender), interventions (herbal preparations, herbal regimen, acupoints selected, frequency, duration and co-interventions if any), controls (type, frequency, and duration), outcomes (measures and time points) and study design (randomisation, allocation concealment, blinding and etc.). If required information was not reported, we tried to request it from the corresponding author of the studies.

\subsection{Risk of bias in individual studies}

The methodological quality of RCTs was assessed according to the risk of bias tool described in the Cochrane Handbook for Systematic Reviews of Interventions. ${ }^{19}$ Six quality elements were assessed: random sequence generation, allocation concealment, blinding of outcome assessors, incomplete outcome data, free of selective reporting, and baseline balance bias.

Study selection, data extraction and risk bias assessment were conducted by two authors (Zhou F, Liang N) independently; in case of discrepancy consensus were reached by discussion with a third party (Liu JP).

\subsection{Data analysis}

Statistical analyses were performed using RevMan 5.3 software. Dichotomous outcomes were analyzed by pooled risk ratio (RR) with $95 \%$ confidence interval (CI) to present effect estimate; Continuous data were presented as mean difference (MD) with 95\% CI. If different measurement scales for continuous data were reported, standardised mean difference (SMD) was performed for data analyses. For the meta-analysis of non-significant heterogeneity, we applied a fixed-effect model (FEM), otherwise, we used randomeffect model for the one of significant heterogeneity ( $F^{2}$ value greater than $\left.50 \%\right) .{ }^{19}$ Subgroup analyses were conducted for different comparisons (SAHP vs no treatment, Sham SAHP, CT, or SAHP plus CT vs CT). Summary of findings table was developed using GRADE pro 3.6.1 software. Funnel plots were generated to detect publication bias if more than 10 trials were identified to report on the same outcome. The results of meta-analyses were visualized in forest plots. 


\section{Results}

\subsection{Study selection}

A total of 1615 records were identified through searching of seven electronic databases.

After excluding 710 duplicates, 905 remaining titles and 244 abstracts were screened and 66 full-text articles were read. The reasons for exclusion during full-text review were "other stage of asthma" ( $\mathrm{n}=19)$, "other outcomes" $(\mathrm{n}=11)$, and "other treatment" $(\mathrm{n}=3)$, "other design" ( $n=2)$. After contacted author, one RCT was excluded due to non randomisation (Fig. 1). Finally, 34 RCTs were included in the descriptive analysis and meta-analysis. ${ }^{20-49}$

\subsection{Characteristics of included RCTs}

All studies originated from and were published in China, of which 23 were journal articles (76.67\%), one was published in conference proceedings $(3.33 \%)^{30}$ and six were dissertations (20\%). $31,36,37,40,41,45$ There were 26 RCTs with two arms, three RCTs with three arms, ${ }^{23,28,39}$ and one trial with two subgroups. ${ }^{36}$

The 34 RCTs involved 3313 participants, varying from $40^{47}$ to $265^{33}$, with a median of 72 participants per trial. Thirteen trials included children exclusively with age from 1 to 14 years old, three trials included adolescents and adults, ${ }^{30,33,44} 13$ trials included adults. One trial did not mention the age of the group. ${ }^{48}$ The mean age of participants ranged from 3.3 to 55.6 years, with a median age of 34.8 years. Regarding gender, $49.3 \%$ participants were female (data not available in two trials). The majority of trials used Chinese national criteria for diagnosis of asthma; only one trial applied the international diagnostic criteria. ${ }^{30}$

Six RCTs described the details of the SAHP which were prepared by the hospital pharmacy $23,25,42,45,47$ or by a pharmaceutical company, ${ }^{38}$ whereas the remaining trials failed to report. The CT used included oral or inhaled corticosteroids, beta2-agonists, leukotriene receptor antagonist, sustained-release theophylline, thymopeptide enteric-coated tablets, and BCG -polysaccharide nucleic acid injection. The duration of treatment ranged from 1 to 3 years, while the treatment frequency ranged from 3 to 9 sessions (Table 1).

Although some of the ingredients of SAHP were not completely the same among the included trials (that might be the main reason of potential heterogeneity), majority of the herbal patches' formulae had same treatment principle, that is, to warm yang and to dispel cold; and the main properties and flavor of the herbs were consistent: acrid-warm and penetrating. Besides, in this review, we took SAHP as a unique TCM therapy and to evaluate its clinical effectiveness rather to look at each herbal formula against its placebo. Hence, we considered SAHP as the therapy in a whole and pooled the data of the same outcome measure from different trials.

None of included studies reported the data collection methods for adverse event. However, based on our clinical observation, we could speculate they evaluate adverse events by regular checks in each $f u$ when participants come back for next session of SAHP. And none of the trials reported the reproducibility of spirometry. In addition, the evaluation time points of each RCT varied from 3 months to 12 months, however, the follow up was at least 6 months since the first session of SAHP finished. 


\subsection{Risk of bias of the included trials}

Although all trials reported "randomisation" in the text, only 12 RCTs described the procedure of randomisation, using random number table $21,26,27,29,33,34,39,41,42,48$ or software. ${ }^{35,45}$ Two RCTs applied opaque envelopes to conceal the allocation. ${ }^{30,45}$ Eight RCTs showed low risk of performance bias by using double blinding, ${ }^{20,21,29,30,33,35,37,45}$ and one RCT used assessor blinding. ${ }^{23}$ Three of 30 RCTs reported the number of dropouts, ${ }^{26,36,39}$ and none of them applied the intention to treat analysis (Fig. 2).

\subsection{Effect estimates}

\subsubsection{Frequency of asthma exacerbations (times per year)-Thirteen RCTs} reported outcome of asthma exacerbation. Two meta-analyses revealed decreased mean frequency of exacerbation from SAHP compared with no intervention (MD: $-1.13 ; 95 \% \mathrm{CI}$ : -2.01 to -0.26 ; FEM; $I^{2}=31 \% ; 2$ RCTs), ${ }^{28,31}$ and SAHP plus CT compared with the same CT (MD: -1.42 ; 95\% CI: -2.19 to -0.65 ; REM; $P^{2}=98 \%$; 7 RCTs). $22,24,36,40,43,46$ Similar effect was found for SAHP when compared with sham SAHP (MD: -1.85 ; 95\%CI: -2.33 to -1.37). ${ }^{21}$ However, a meta-analysis showed no significant difference between SAHP and CT (MD: -0.49 ; 95\%CI: -1.27 to 0.29 ; REM; $P^{2}=94 \% ; 3$ RCTs) (Supplementary Fig. S1 in the online version at DOI: http://dx.doi.org/10.1016/j.ctim.2016.11.007). ${ }^{28,38,44}$ Xie's RCT compared SAHP with thymopeptide enteric-coated tablets found statistical difference on frequency of asthma exacerbations favouring SAHP (MD: -1.39 ; $95 \%$ CI: -1.96 to $-0.82) .{ }^{42}$

One RCT reported the asthma exacerbation recurrence rate, showing improvement from SAHP compared with sham SAHP (MD: 0.42; 95\% CI: 0.26-0.69). ${ }^{33}$ Another RCT, comparing SAHP with no treatment, did not find a significant difference for the duration of asthma exacerbation (MD: 35.76 min.; 95\% CI: -49.57 to 121.09). ${ }^{30}$

3.4.2. Asthmatic symptom improvement (scores)—Eight RCTs evaluated the mean scores in asthmatic symptoms. A meta-analysis found no significant difference between real and sham SAHP (SMD: -0.94 ; 95\%CI: -2.36 to 0.48 ; REM; $I^{2}=95 \%$; 3 RCTs), ${ }^{21,37,45}$ while the other meta-analyses found beneficial effects of SAHP compared with CT (SMD: -0.43 ; 95\%CI: -0.71 to -0.16 ; FEM; $I^{2}=0 \%$; 3 RCTs). ${ }^{34,41,49}$ Similar for the effects of SAHP plus CT compared with CT alone (SMD: -1.22 ; $95 \%$ CI: -1.55 to -0.88 ; REM; $P^{2}=$ $0 \% ; 2$ RCTs). ${ }^{26,48}$ Because of reverse direction of evaluation scores, one RCT was not included into the meta-analysis and showed no effect of SAHP compared with sham SAHP (MD: 0.43 ; 95\%CI: -0.16 to 1.02 ) (Supplementary Fig. S2 in the online version at DOI: http://dx.doi.org/10.1016/j.ctim.2016.11.007). ${ }^{45}$

\subsection{Lungfunction}

Compared with sham SAHP, Shi' RCT showed beneficial effect on PEF\% (MD: 11.23; 95\%CI: 4.04-18.42). ${ }^{35}$ However, when compared with CT, Hu's RCT did not find statistical difference on PEF (MD: $-11.94 \mathrm{~L} / \mathrm{min}$.; 95\% CI: -36.17 to 12.29$){ }^{23}$

One meta-analysis, comparing SAHP with sham SAHP, found a beneficial effect on PEF (MD:35.37 L/min; 95\%CI: 1.03 to 69.71; REM; $I^{2}=79 \% ; 2$ RCTs); ${ }^{23,33}$ while the other two 
meta-analyses with the same comparison showed no significant difference on $\mathrm{FEV}_{1} \%$ (MD: 2.45; 95\%CI: -16.72 to 21.61 ; REM; $I^{2}=89 \%$; 2 RCTs) ${ }^{35,45}$ and on $\mathrm{FEV}_{1} / \mathrm{FVC}$ (MD: 7.29; 95\%CI: -7.29 to 22.37 ; REM; $P=93 \%$; 3 RCTs) ${ }^{29,35,45}$ (Supplementary Figs. S3-S6 online version at DOI: http://dx.doi.org/10.1016/j.ctim.2016.11.007).

Comparing SAHP with CT, one single RCT and two meta-analyses showed no statistical difference on $\mathrm{FEV}_{1} / \mathrm{FVC}$ (MD: -2.88 ; 95\%CI: -13.07 to 7.31 ) ${ }^{39}$; while two meta-analyses showed no statistical difference on PEF\% (MD: 2.02 ; 95\%CI: -6.52 to 10.56 ; REM; $P^{2}=$ $64 \% ; 2 \mathrm{RCTs}$ ), ${ }^{22,23,39}$ and $\mathrm{FEV}_{1} \%$ (MD: $4.11 ; 95 \% \mathrm{CI}$ : -0.41 to $8.63 ; 2 \mathrm{RCTs}$ ). ${ }^{22,39}$

Comparing SAHP plus CT with CT alone, two meta-analyses and a single trial revealed effects on PEF\% (MD: 8.74; 95\%CI: 3.53-13.96; REM; $P^{2}=98 \%$; 2 RCTs), ${ }^{39,49} \mathrm{FEV}_{1} \%$ (MD: 8.57; 95\%CI: 5.01-12.13; REM; $I^{2}=97 \%$; 3 RCTs), ${ }^{36,39,48}$ and $\mathrm{FEV}_{1} / \mathrm{FVC}$ (MD: 11.68; 95\%CI: $1.41-21.95) .{ }^{39}$

3.5.1. Quality of life (scores)—Three RCTs reported on quality of life using Asthma Quality of Life Questionnaire (AQLQ) ${ }^{50}$ A Meta-analysis showed that SAHP was better than sham SAHP(MD: 6.45 ; $95 \%$ CI: 3.42 to 9.48; FEM; $P^{2}=0 \%$; 2 RCTs) ${ }^{29,45}$ Shen's trial compared SAHP with CT, showing better effect from SAHP (MD: 13.66; 95\% CI: 2.91 to $24.41)^{34}$ (Supplementary Fig. S7 in the online version at DOI: http://dx.doi.org/10.1016/ j.ctim.2016.11.007).

A summary of finding table presented the main findings of SAHP plus CT versus CT for stable asthma, and provided key information about the quality of evidence, and a summary of important statistical results on mean frequency of asthma exacerbations, asthmatic symptoms and lung function outcomes (Table 2).

\subsection{Safety}

Of 30 trials, only 9 trials (30\%) reported on adverse events: two of them reported that no adverse events occurred ${ }^{20,25}$ While seven trials demonstrated that adverse events were mild, and well tolerated, including skin itch $\left(11 / 202\right.$ in 5 trials $\left.^{37,38,40,41,47}\right)$, skin blister (1/31, $3.23 \%)^{40}$ and allergy $(1 / 30,3.33 \%)^{27}$

\section{Discussion}

\subsection{Summary of main findings}

Thirty four RCTs were included in this review, SAHP as adjuvant therapy with CT seems to be more effective than CT alone for alleviating asthma on both subjective and objective outcomes. Compared with sham SAHP, SAHP alone seems to be effective for preventing asthma exacerbation, and for improving lung function and quality of life SAHP as adjuvant therapy with CT seems to be more effective than CT alone for alleviating asthma on both subjective and objective outcomes. Compared with sham SAHP, SAHP alone seems to be effective for preventing asthma exacerbation, and for improving lung function and quality of life. However, we could not draw conclusion due to the included studies with high or unclear risk of bias. To evaluate safety of SAHP, the more wide study inclusion criteria (including other study designs) is desirable. Hence, although no serious adverse events were reported in 
included RCTs, we could not reach firm conclusion on its safety due to lack of evidence from non-randomised studies.

\subsection{Comparison with previous systematic review}

The previously published systematic review, which focused on AHP (no matter during sanfu or not) versus any comparator without AHP, found evidence that AHP improves the immune outcomes for childhood asthma on the basis of 6 RCTs published up to $2014^{16}$; The other one only involved one Chinese database and included 16 RCTs, found encouraging and not conclusive evidence of the AHP (also no matter during sanfu or not) efficacy on pulmonary functions and asthmatic symptoms, however, this systematic review missed to evaluated the prevention effectiveness of AHP on asthma and quality of life. ${ }^{17}$

\subsection{Internal and external validity}

In general, the risk of bias in the included studies was high or unclear. First, allocation concealment is a valid measure to avoid selection bias. However, there were only two RCTs which reported on allocation concealment. Further, the chances for performance bias and detection bias would increase because $73.53 \%$ RCTs did not apply blind to participants and personnel. Last, few trials provided adequate information on incomplete outcome data, which may contribute to potential attrition bias. Therefore, the potential for various biases strongly limit the interpretability of results.

Participants involved in this systematic review have three characteristics, 1) all the 3313 asthma patients were from China, 2) patients' age range covered children, adolescents, adults and elderly people, and 3) the sex ratio was almost half to half. Hence, this diversity shows that SAHP has a good applicability and acceptance rate in the real world at least in China. However, for other ethnicities different from Chinese, the effectiveness of SAHP is still unknown. In addition, all included trials did not mention co-interventions, the main reason might be the purpose of stable asthma treatment is mainly prophylactic.

\subsection{Strengths and weaknesses}

This review, to the best of our knowledge, is the first one comparing SAHP plus CT versus $\mathrm{CT}$ alone and evaluating the preventive and therapeutic effectiveness of SAHP on stable asthma. The strengths of this review mainly include the comprehensive literature search without language restrictions, the high number of RCTs involving children $(56.67 \%, 17 / 30)$ and the subgroup analyses to assess the SAHP's effects in different combinations and scenarios. On the other hand, the poor methodological quality of the studies included is one of the limitations in this review. Secondly, all included studies were conducted in China, which restricts the generalizability of the findings due to the context in terms of tradition and culture. Lastly, most of the meta-analyses in this review showed high heterogeneity. Heterogeneity might be partly related to differences of the acupoints selected, the SAHP formula selected and various conventional treatments applied. Although this limits our quantitative analyses, it also reflects asthma management in the real clinical world in China. 


\subsection{Implications for clinical practice}

Compared with sham SAHP, real SAHP alone seems to be effective for preventing asthma exacerbation, and for improving lung function and quality of life. This could carefully be interpreted as a hint on the therapeutic effects of SAHP alone. Furthermore, the similar effects of SAHP and CT interventions could be cautiously interpreted as the effectiveness of SAHP alone.

For the SAHP intervention, the most critical aspects are the herbal patching's regimen and the acupoints selected. Based on our review, the four major herbs of the patching regimens are Semen Sinapis, Rhizoma Corydalis, Radix et Rhizoma Asari, Radix Kansui, while the basic four acupoints are Tian tu (CV 22), Dan zhong (CV 17), Da zhui (GV 14), Fei shu (BL 13). Until now, there are some pre-clinical studies reported that using these herb on these acupoints could regulate the immune mechanism and decrease the inflammation. ${ }^{51-53}$ However, considering the high risk of bias all the conclusions should be interpreted with caution.

\subsection{Implications for further research}

The use of rigorous research methods, including pretrial sample size calculation, appropriate randomisation, strict allocation concealment, blinding at least for outcome assessors and statisticians, and intention-to-treat analysis, should be ensured in future RCTs. Scientific reporting according to the consolidated standards of reporting trials (CONSORT) statement ${ }^{54}$ is also critical for future RCTs.

In addition, it is worth noting that the sham SAHP used in the included studies seems to be easily recognized by both physicians and patients. A perfect sham SAHP should look exactly like true SAHP. A sham SAHP without herbs is unlikely to simulate the same color and smell as true SAHP. Hence, the preparation of good sham SAHP is also a challenge for future RCTs.

\section{Conclusion}

SAHP alone or combined with CT appears to be more effective than sham SAHP or CT on reduction of asthma exacerbations, improving lung function, and SAHP seems to be safe. However, the findings should be interpreted with caution due to limitations in trial methodological quality. Further, rigorously designed, large-scale trials are warranted for stronger evidence.

\section{Supplementary Material}

Refer to Web version on PubMed Central for supplementary material.

\section{Acknowledgments}

Fen Zhou was supported by the program for Young Talents Plan of Beijing Municipal Education Commission (2013-YETP-0796). This work is supported by the Program of Research Capacity Building from the State Administration of Traditional Chinese Medicine (No. 201207012), and the 111 Project (B08006). Jianping Liu was partially funded by Grant Number R24AT001293 from the National Center for Complementary and Alternative 
Medicine of the US National Institutes of Health. Thanks for EURASIA-PACIFIC UNINET's scholarship to F Zhou.

\section{References}

1. FitzGerald J. Global strategy for asthma management and prevention. Glob Initiat Asthma. 2015

2. To T, Stanojevic S, Moores G, et al. Global asthma prevalence in adults: findings from the crosssectional world health survey. BMC Public Health. 2012; 12:204. [PubMed: 22429515]

3. Anandan C, Nurmatov U, van Schayck OCP, Sheikh A. Is the prevalence of asthma declining? Systematic review of epidemiological studies. Allergy. 2010; 65:152-167. [PubMed: 19912154]

4. Rassool, GH. The World Health Report 2004-Changing History. World Health Organization; p. 2004542

5. Chipps BE, Zeiger RS, Dorenbaum A, et al. Assessment of asthma control and asthma exacerbations in the epidemiology and natural history of asthma: outcomes and treatment regimens (TENOR) observational cohort. Curr Respir Care Rep. 2012; 1:259-269. [PubMed: 23136642]

6. Miller MK, Lee JH, Miller DP, Wenzel SE. Recent asthma exacerbations: a key predictor of future exacerbations. Respir Med. 2007; 101:481-489. [PubMed: 16914299]

7. Alvarez GG, Schulzer M, Jung D. Fitzgerald JM: A systematic review of risk factors associated with near-fatal and fatal asthma. Can Respir J. 2005; 12:265-270. [PubMed: 16107915]

8. Price D, Bosnic-Anticevich S, Briggs A, et al. Inhaler competence in asthma: common errors, barriers to use and recommended solutions. Respir Med. 2013; 107:37-46. [PubMed: 23098685]

9. Lindsay JT, Heaney LG. Nonadherence in difficult asthma-facts, myths, and a time to act. Patient Preference and Adherence. 2013:329-336. [PubMed: 23723690]

10. Zhang, L. Zhangshi yi tong. Shanghai: Shanghai Science Technology Publications; 1990.

11. Zhang, Z., Huang, Y., Tao, J., Li, Z. Internal Medicine of Traditional Chinese Medicine. Shanghai: Publishing house of shanghai university of traditional Chinese medicine; 2006.

12. Lorraine W. San Fu moxibustion and lung-related disorders. J Chin Med. 2009; 1:4-18.

13. Wang Q, Dong L, Liu JP, Jiang D. Transdermal treatment with Chinese herbal medicine: theory and clinical applications. Science. 2015 in press.

14. Mellinkoff SM. Yin and yang. Arch Intern Med. 1968; 122:81. [PubMed: 5659383]

15. Cai JX, YDLM. Effect of KeChuanSanFuTie on IL-4, IFN- $\gamma$ in children patients in the asthma remission. Liaoning J Tradit Chin Med. 2010:485-487.

16. Yang XC, Yin T, Gao Q, Kong LJ. The immunomodulatory effect of acupoint application for childhood asthma: a systematic review and meta-Analysis. Evid Based Comp Altern Med. 2015; 2015:1-8.

17. Lee SH, Chang GT, Zhang X, Lee H. Acupoint herbal patching for asthma: a systematic review and meta-analysis of randomized controlled trials. Medicine (Baltimore). 2016; 95:e2439. [PubMed: 26765427]

18. Moher D, Liberati A, Tetzlaff J, Altman DG. preferred reporting items for systematic reviews and meta-Analyses: the PRISMA statement. Ann Intern Med. 2009; 151:264-269. [PubMed: 19622511]

19. HJG, S. Cochrane Handbook for Systematic Reviews of Interventions. John Wiley \& Sons Ltd; 2008.

20. Cai JX, Chen JH, Chen H, Zhang YX. Clinical effectiveness of sanfu patching for preventing and treating children asthma. Clin J Tradit Chin Med. 2010; 22:485-487.

21. Chang JJ. Clinical research of asthma patching during sanfu days for preventing cold type asthma. Res Integr Tradit Chin West Med. 2013; 5:5-8.

22. Deng YP. Sanfu patching for children asthma in remission stage on lung function. Hubei J TCM. 2012; 34:19-20.

23. Hu HP, Luo F, Zhang JY, et al. Clinical observe of prevent and treat children asthma using drugs at the umbilical region. J Pediatr Tradit Chin Med. 2005; 1:29-34.

24. Jin P, Fang J, Zhou F. United acupoint application in bronchial asthma inhaled cellular immune function of children. China Modern Dr. 2011; 49(4-5):14. 
25. GP, Li. Clinical effectiveness observation of sanfu patching for preventing and treating children variant asthma. Med Front. 2012:100-101.

26. Li HY, Zhang Y, Zhong XJ, et al. Clinical observation of acupoint patching combined with direct current iontophoresis for bronchial asthma in remission stage. Liaoning J Tradit Chin Med. 2015; (05):1067-1071.

27. Li XY, Yuan XF. Clinical research of sanfu patching for asthma in remission stage on immunoregulation function. China Pract Med. 2010; 5:128-129.

28. Liu JZ, Gong HW, Peng YH, Liu CY. External patching of Chun Fu Ling for preventing children asthma in 30 cases. Chin Arch Tradit Chin Med. 2003; 21:1958-1959.

29. Lu YM. 35 cases of sanfu patching for bronchial asthma in remission stage. Hunan J Tradit Chin Med. 2011; 27:17-19.

30. Luo, GQ., Zhang, W., Li, L., Wang, ZH. Randomised double-blinded controlled trial of sanfu moxibustion for non-acute bronchial asthma; The 2011 National Conference on Chronobiological Medicine; 2011. p. 225-230.

31. Luo, M. Master's Thesis. Guangxi University of Chinese Medicine; 2008. Effectiveness Research of Sanfu Acupoint Patching for Asthma at Remission Stage with Lung Qi Deficiency.

32. Luo M, Zhang H, Weng H. Clinical observation of 60 cases of acupoint patching on sanfu period for bronchial asthma in remission stage. Yunnan J Tradit Chin Med Mater Med. 2007:26-27.

33. Lv JB, Ke WM, Zhou XH, He H, Wu JQ, Chen CM. Standardization research on crude herb moxibustion for treating bronchial asthma. Shanghai J Acupunct Moxib. 2014:1013-1015.

34. Shen JY, Ni W, Zhang WQ, Jiang Y. Clinical research of shao's asthma patching for bronchial asthma with yang deficiency. J Sichuan Tradit Chin Med. 2014:170-172.

35. Shi LJ, Zhao Y. Clinical observation of acupoint patching in summer for winter diseases therapy for preventing children asthma recurrence in 92 Cases. J Emerg Tradit Chin Med. 2014; 23:23422344.

36. Wang, SH. Master's Thesis. Fujian University of Chinese Medicine; 2008. Observe the Clinical Effectiveness of Baijiezi Pill Rreat Asthma by Stick to Acupuncture Points in Hot Summer Days and Study Its Action on IL-13, IFN- $\gamma$ in Asthma.

37. Wang, XW. Master's Thesis. Heilongjiang University of Traditional Chinese Medicine; 2013. Effectiveness Comparison of sanfu Acupoint Patching with Moxibustion for Treating Bronchial Asthma.

38. Wang YF. Clinical observation of acupoint patching for children asthma in remission stage in 90 cases. J Zhejiang Chin Med Univ. 2012; 36:79-81.

39. Wu F, Yao MH, Zhu Y. Clinical study on prevention of recurrence of asthma in children by Xiaochuangao acupoint paste: treating winter diseases in summer. China J Chin Mater Med. 2012; 37:2646-2648.

40. JH, Wu. Master's Thesis. Shandong University of Traditional Chinese Medicine; 2011. Clinical Research of Acupoint Patching for Children Asthma in Remission Stage with Lung-kidney Deficiency.

41. Xie, MM. Master's Thesis. Hubei University of Chinese Medicine; 2008. Clinical Observation of sanfu Acupoint Patching for Adult Asthma.

42. Xie ZW, Shi FH. Effectiveness analysis of acupoint patching for asthma. Shaanxi J Tradit Chin Med. 2014; 36:3-364.

43. Xiong YH. Clinical research of treating winter-diseases in summer acupoint application therapy in the treatment of children with remission stage asthma. Clin Med Eng. 2014; 21:481-482.

44. Yang L, Cao Y. Clinical effectiveness evaluation of acupoint patching combined with traditional Chinese medicine care for treatment of bronchial asthma in remission stage. Guangming J Chin Med. 2014; 29:602-603.

45. DD, Yu. Master's Thesis. Guangzhou Medical College; 2009. Clinical Study of Efficiency of Bronchial Asthma Treated with Acupoint Application Therapy.

46. Yu YL, Shang LL. Clinical observation of treatment with point application to bronchial asthma of children in remission. Guangming J Chin Med. 2011; 26:978-981. 
47. Zhao Y, Li GQ, Li H. 21 Cases of ccupoint patching of Xiaochuangao for preventing asthma progress in winter. J Tradit Chin Med. 2012; 53:784-786.

48. Zhu JF, You JS, Shi PY, et al. Clinical observation of Wu's cream on acupoints combined with inhaled seretide for bronchial asthma. J New Chin Med. 2011:108-110.

49. Zhu XM, Chen X. Clinical observation on 72 cases with bronchial asthma treated with Huagaitie. Gansu J Tradit Chin Med. 2012; 25:75-77.

50. Juniper EF, Guyatt GH, Willan A, Griffith LE. Determining a minimal important change in a disease-specific Quality of Life Questionnaire. J Clin Epidemiol. 1994; 47:81-87. [PubMed: 8283197]

51. Xu HJ. The review on herbal patching for bronchial asthma immune system. World J Integr Tradit West Med. 2015; 10:1761-1763.

52. He Q, Zhang L, Hu XL. Sanjiutie herbal patching on IgE level of asthma rat model. J Xinjiang Med Univ. 2013; 36:200-203.

53. Chen XQ, Xu Y, Ni W, et al. The mechanism study of Yangxuxiaochuanfutiefang herbal patching on bronchial asthma immune system. J Emerg Tradit Chin Med. 2012; 21:701-702.

54. Begg C, Cho M, Eastwood S, et al. Improving the quality of reporting of randomized controlled trials. The CONSORT statement. JAMA. 1996; 276:637-639. [PubMed: 8773637] 

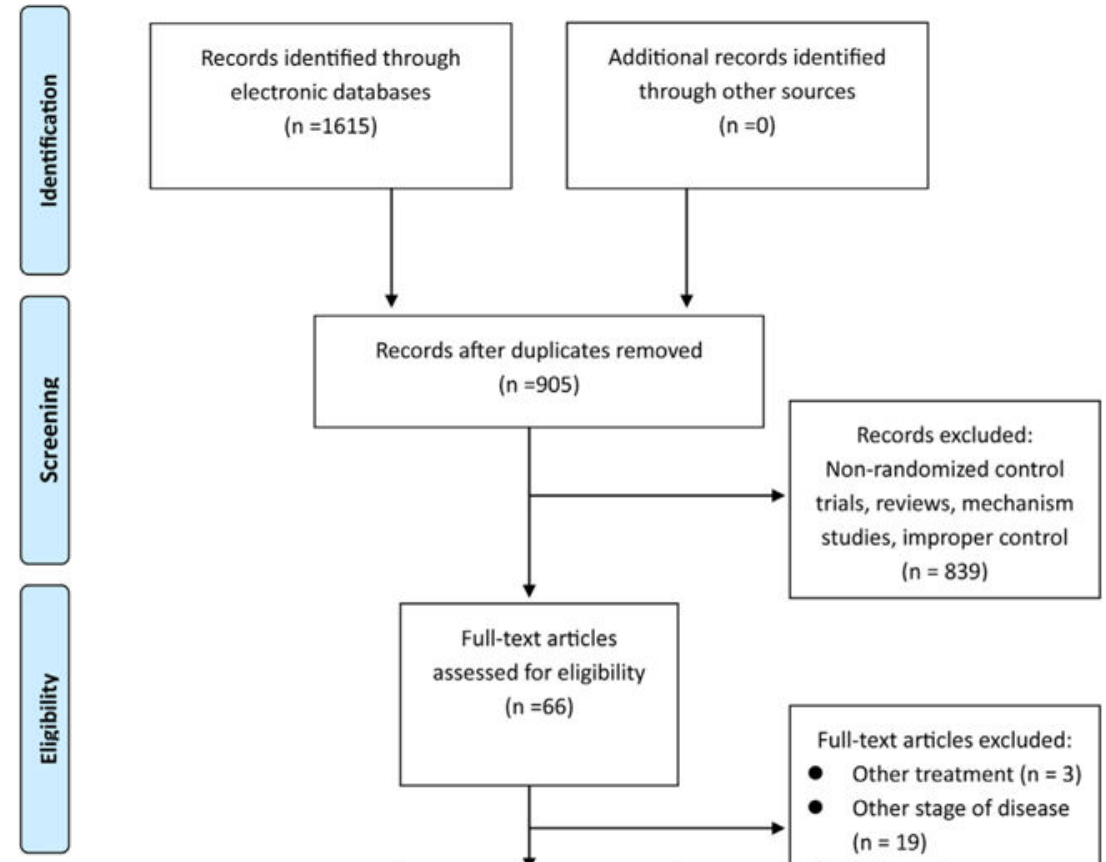

assessed for eligibility ( $n=66)$

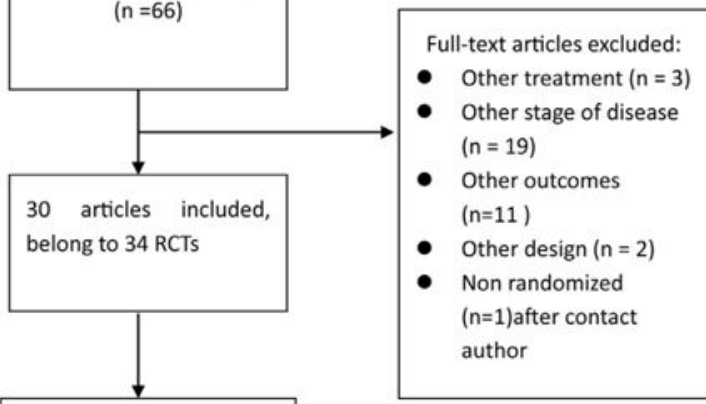

34 RCTs included in quantitative synthesis

Fig. 1.

Flow diagram of study searches and selection. 


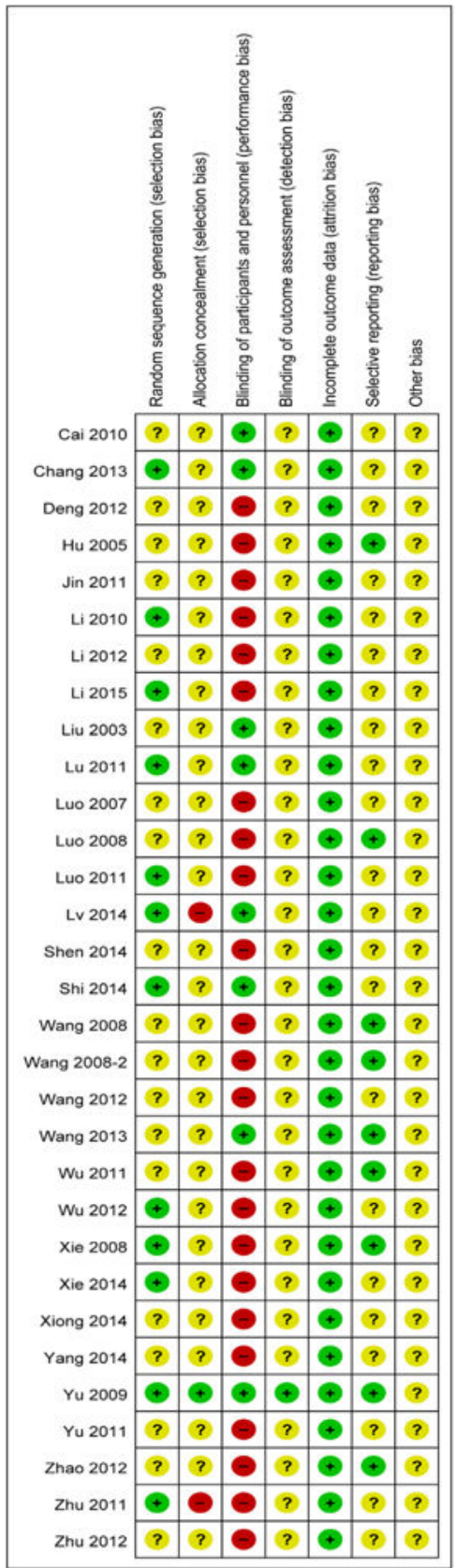

๑Low risk $\boldsymbol{\ominus}_{\text {High risk }}{ }^{\text {? Unclear }}$

Fig. 2.

Risk of bias summary. Presentation of the risk of bias summary of the review author's judgments about each risk of bias item for each included trials. 


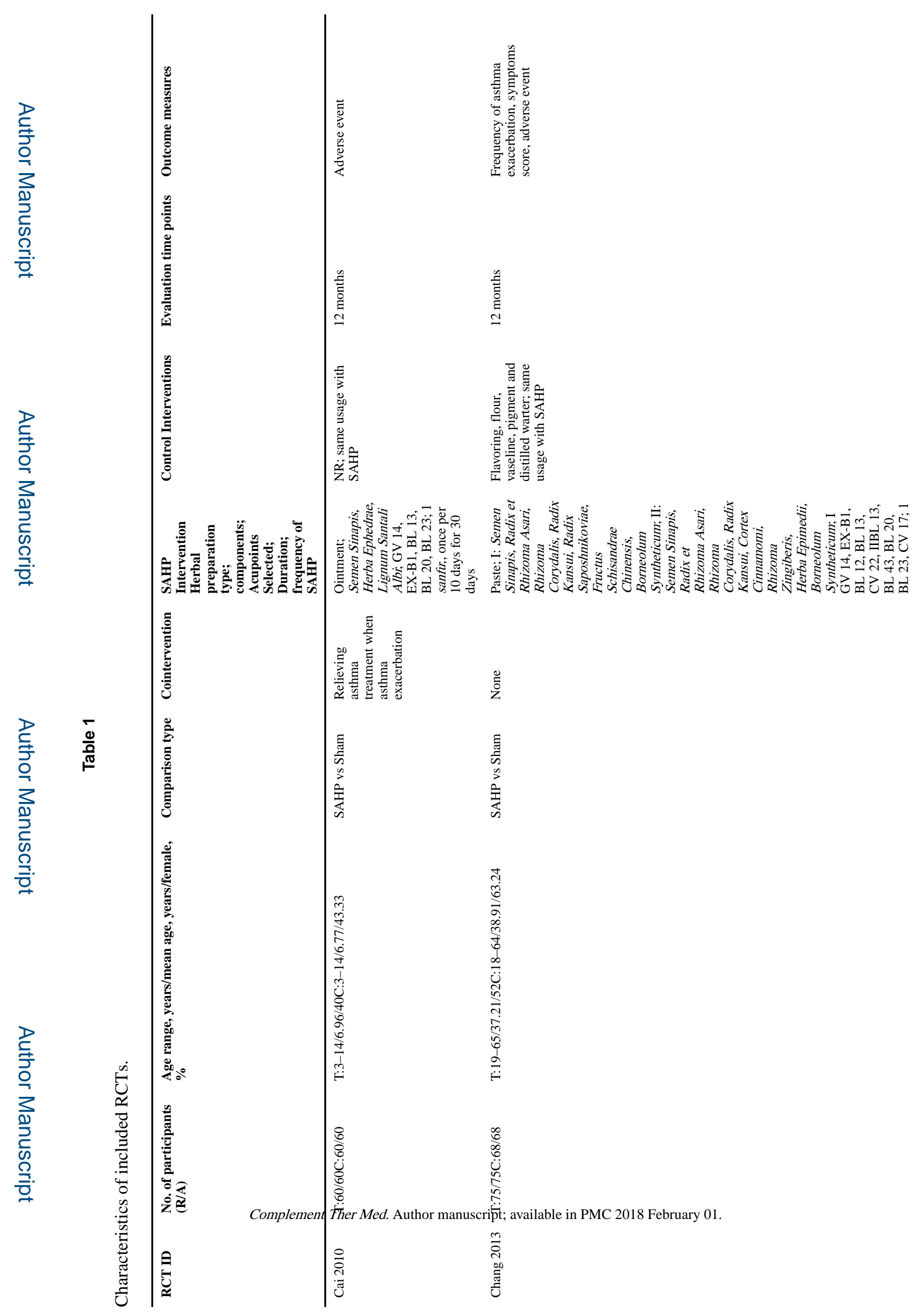




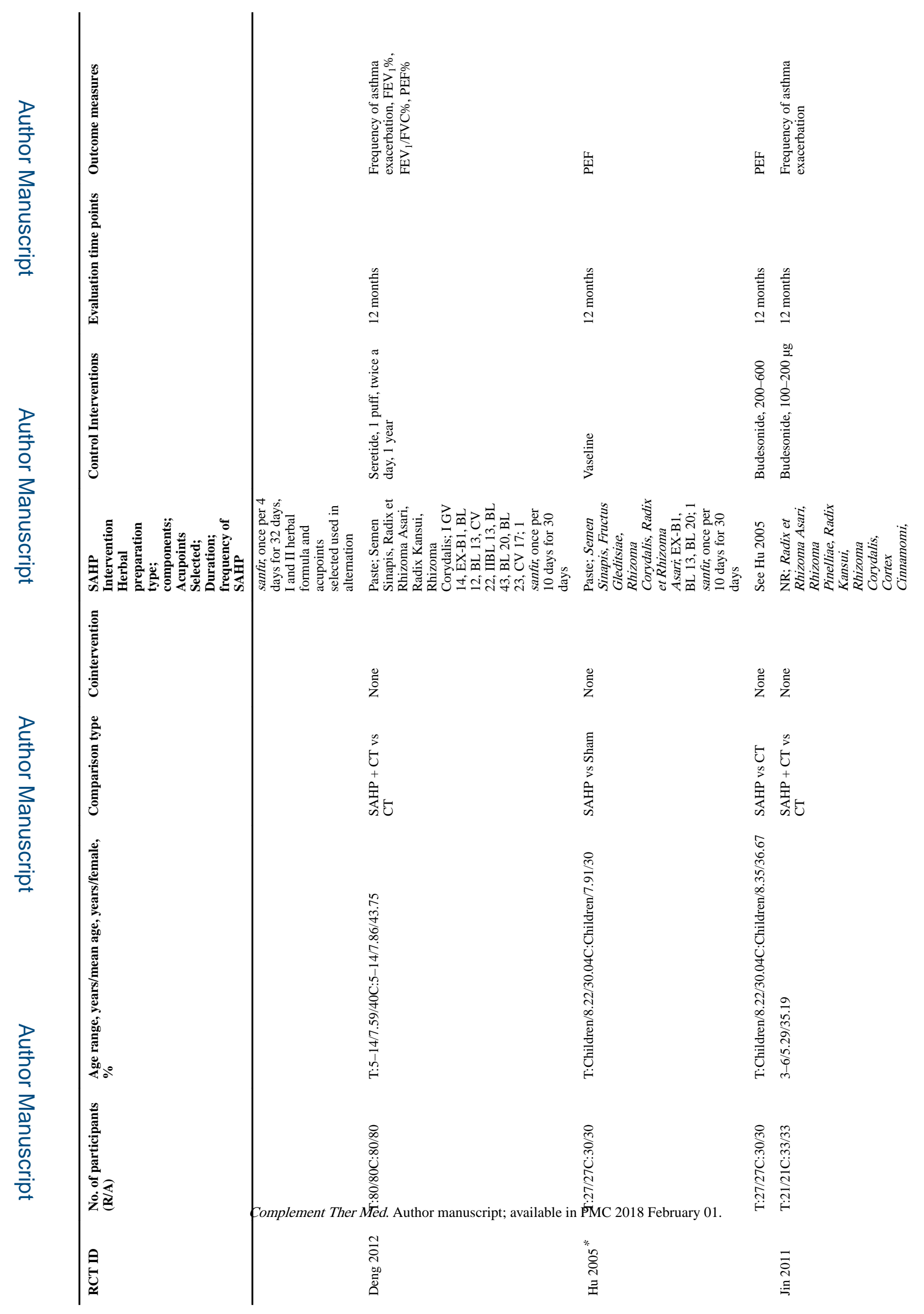




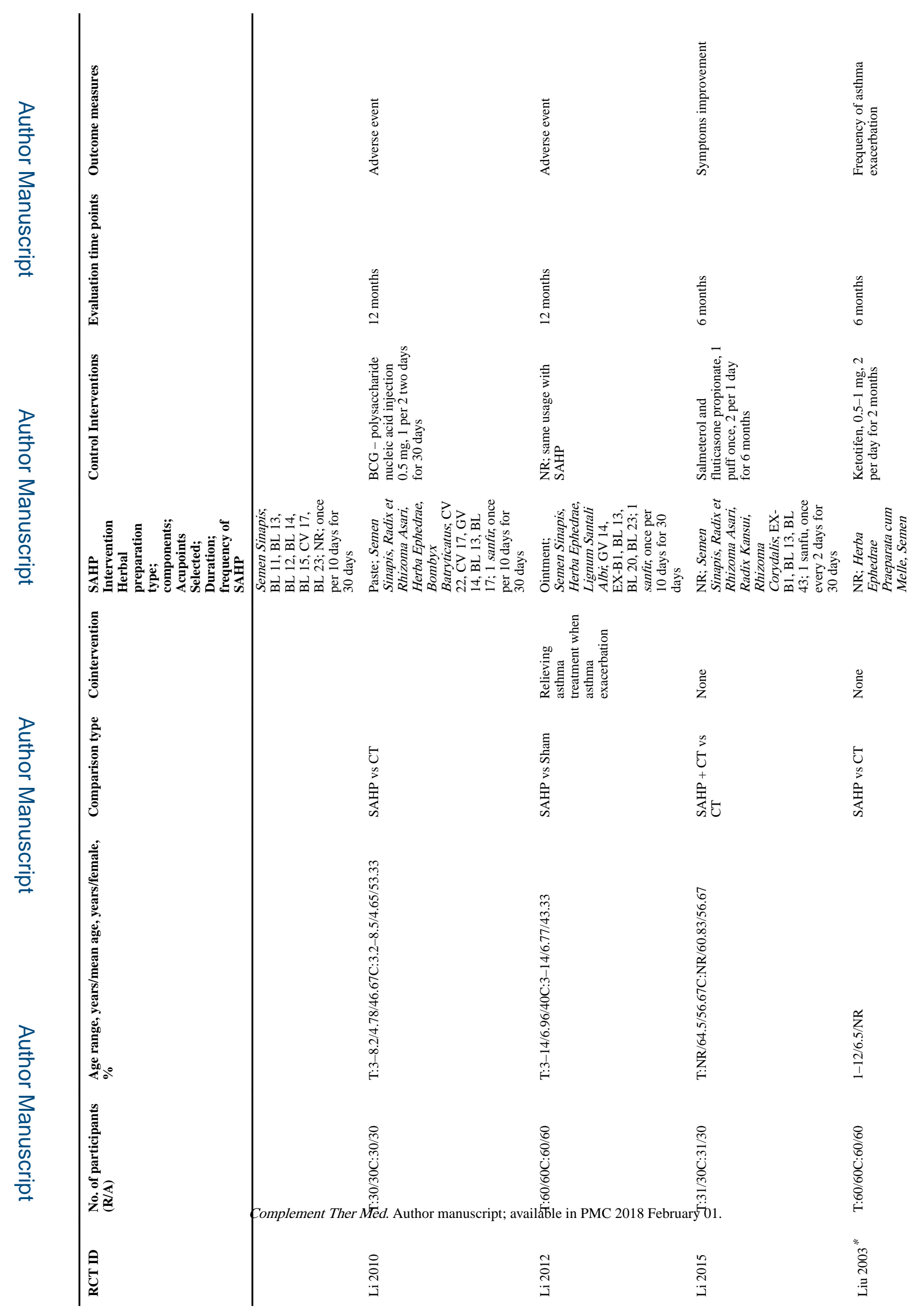




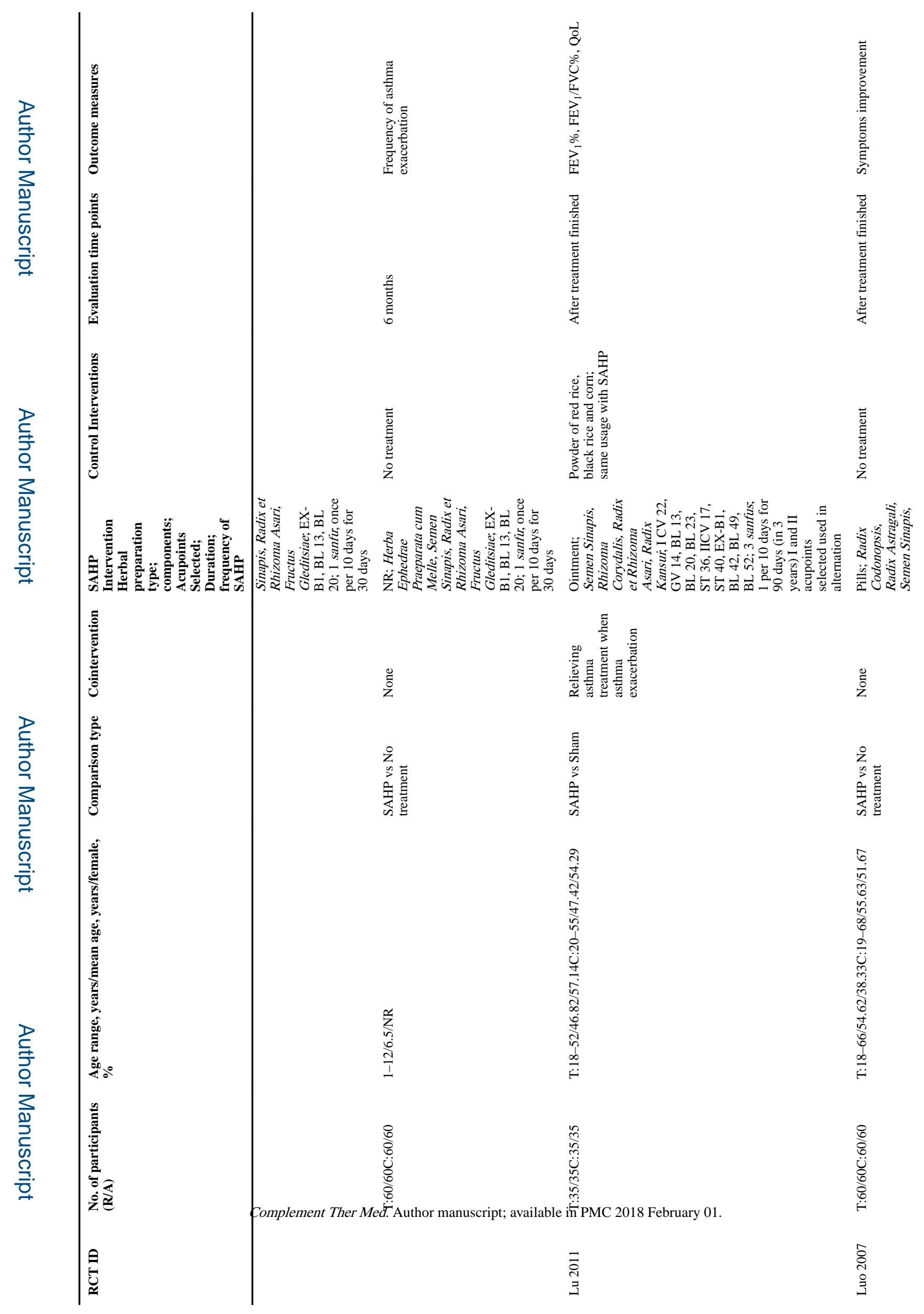




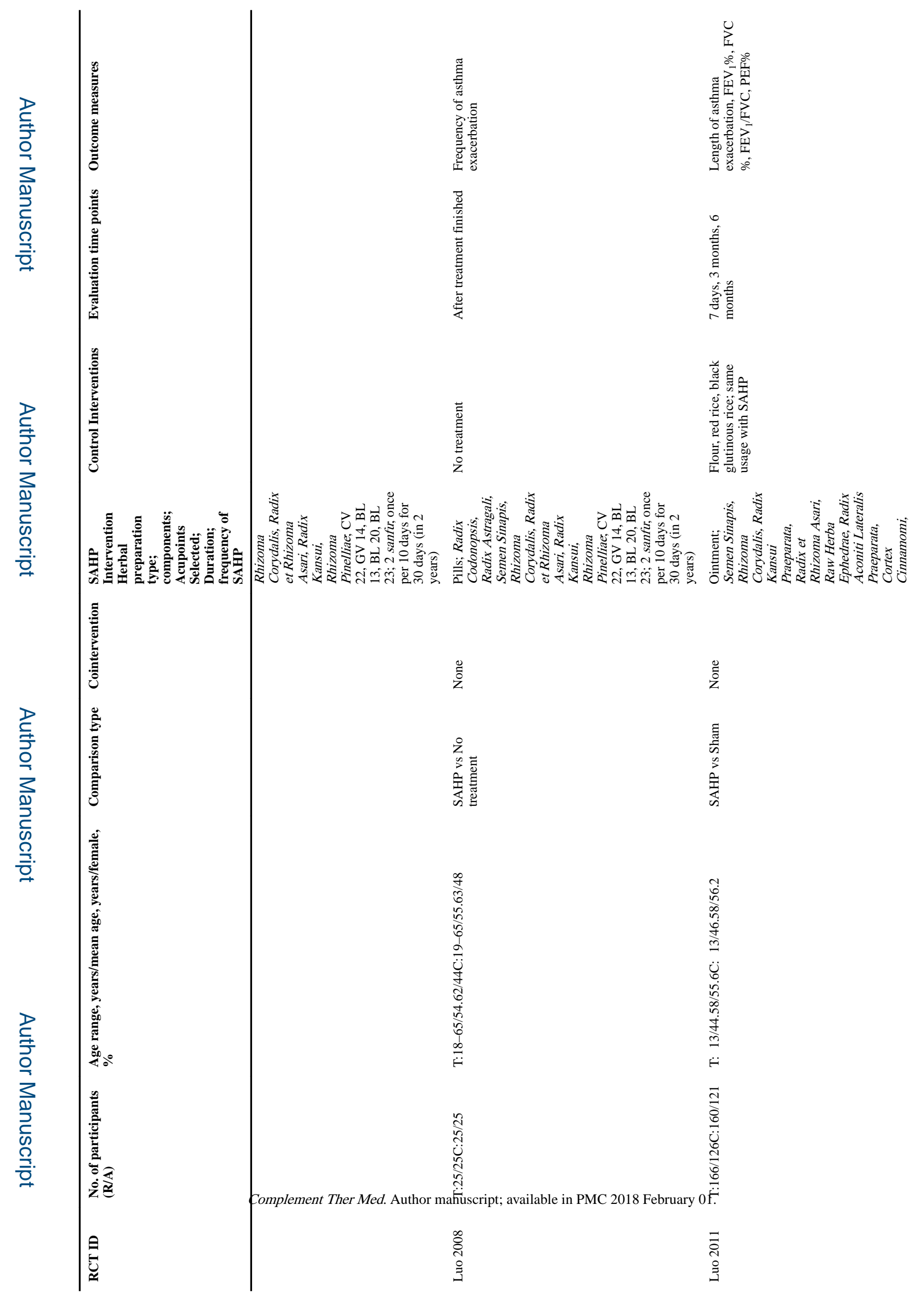




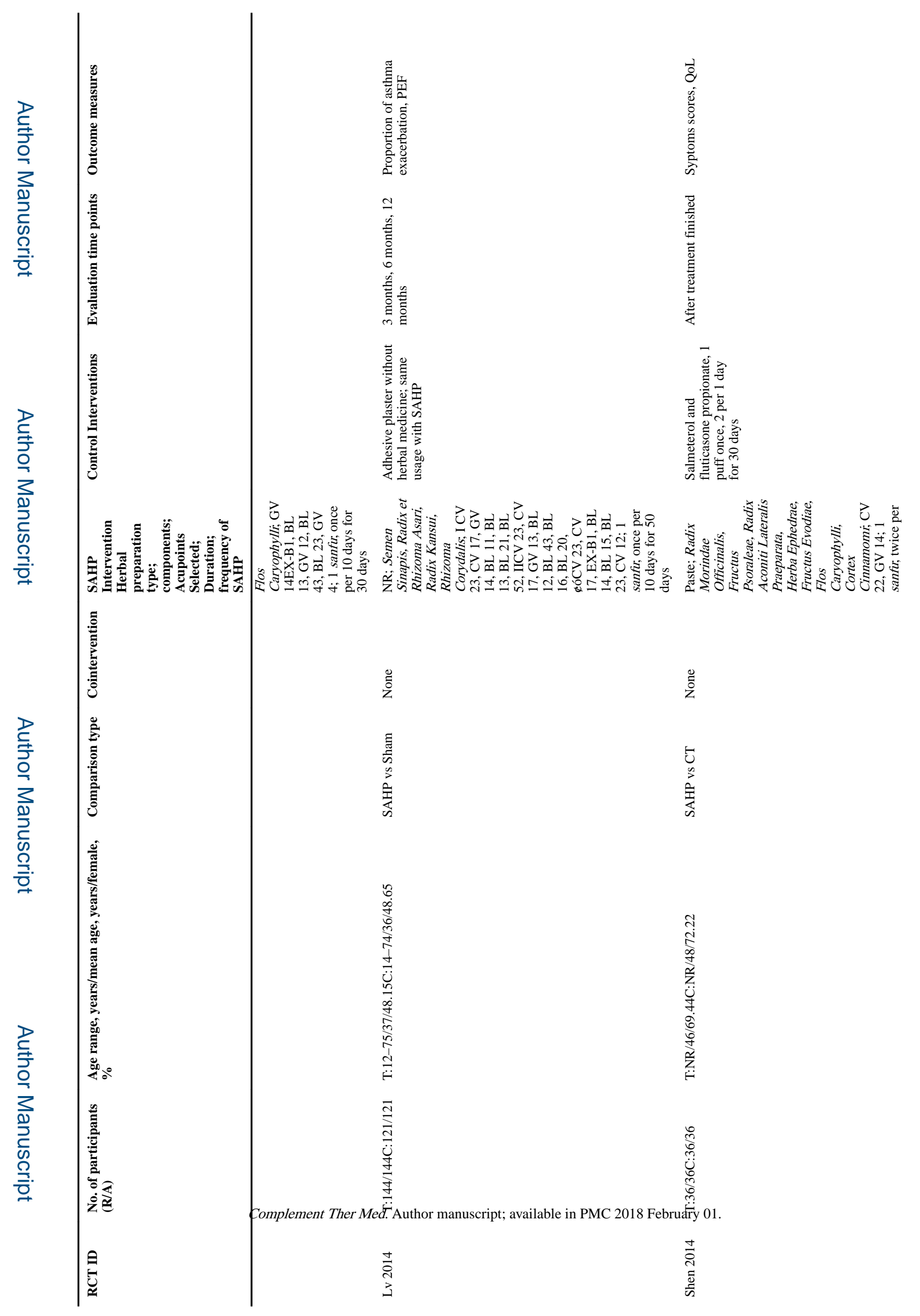




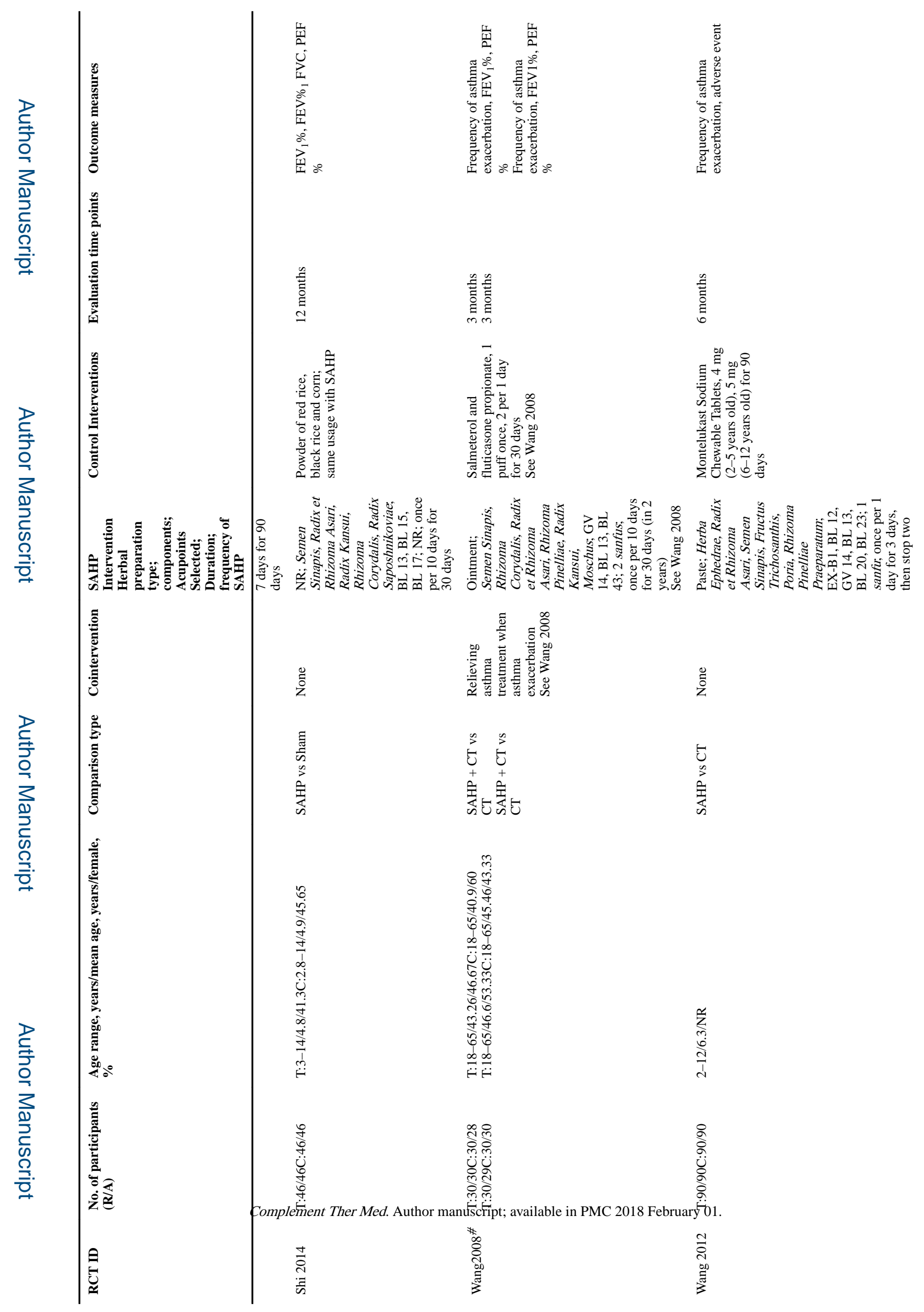




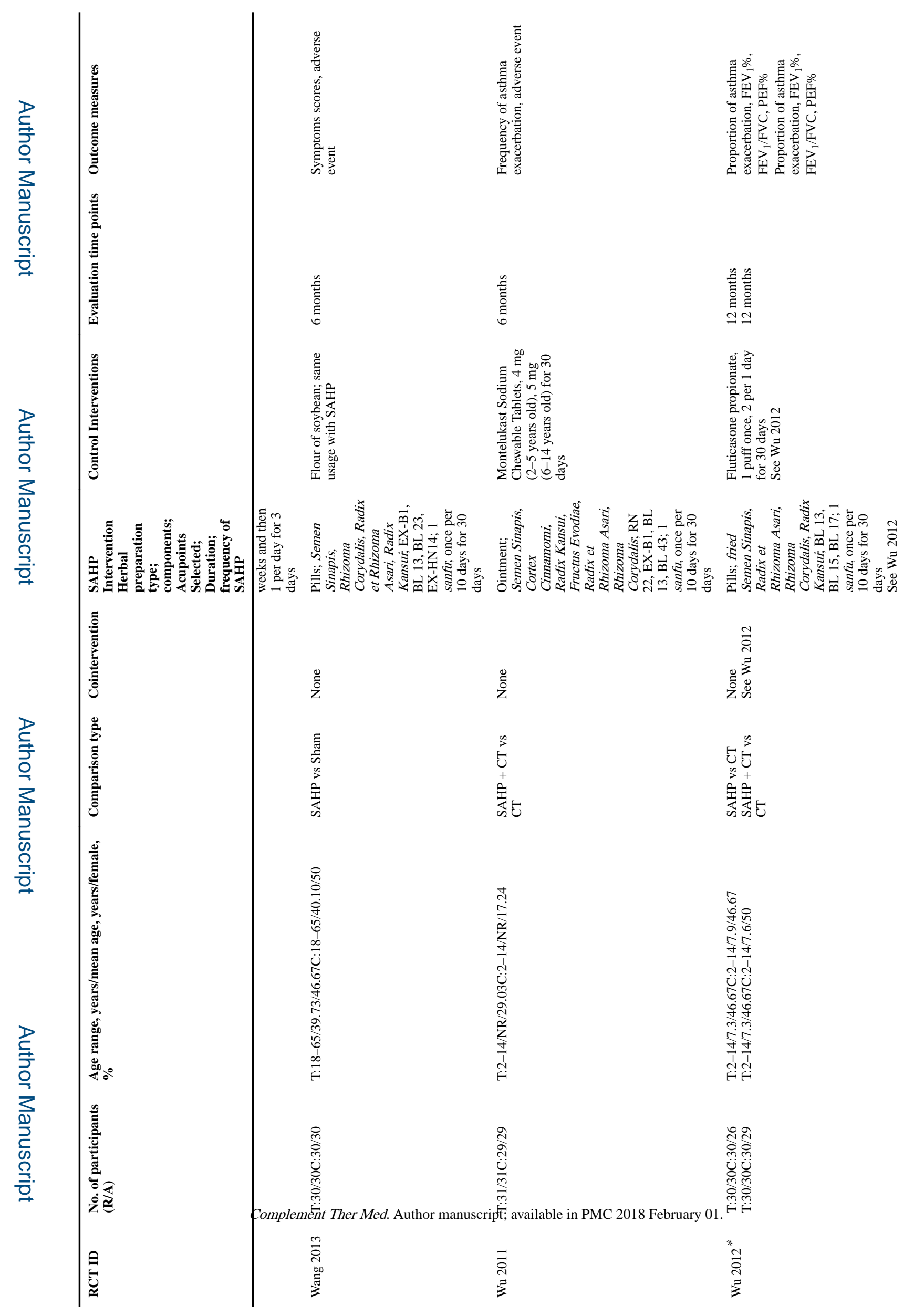




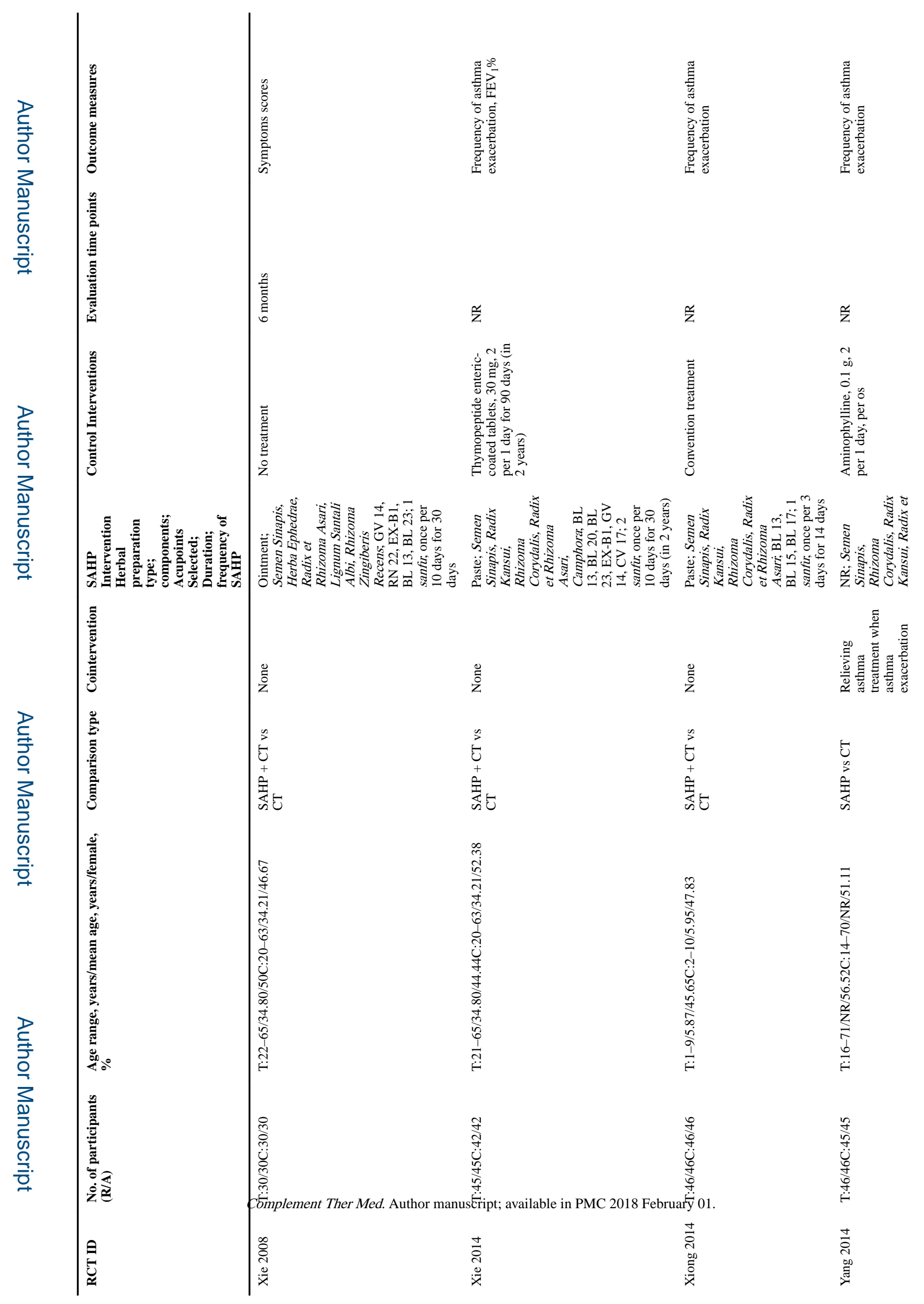




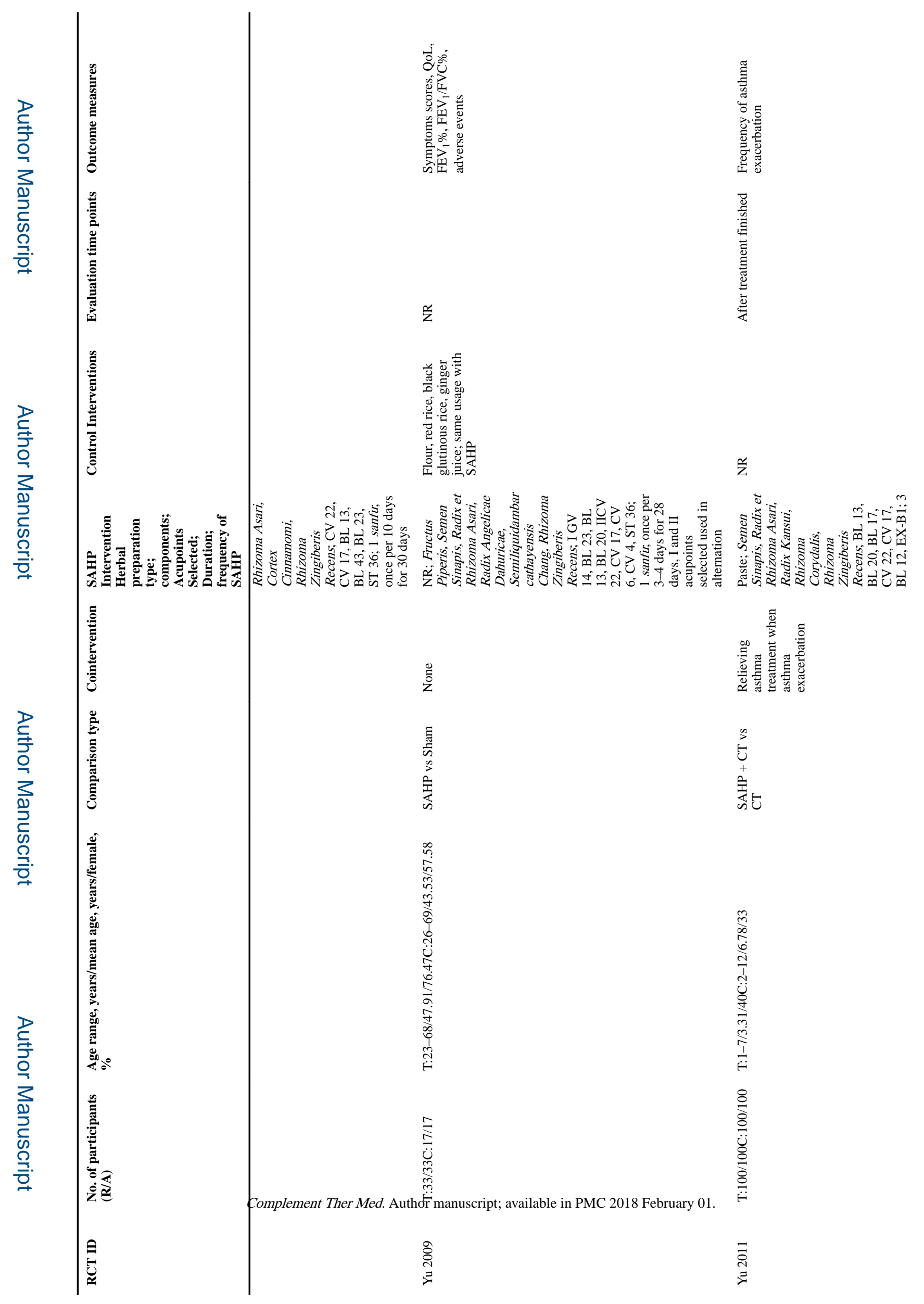




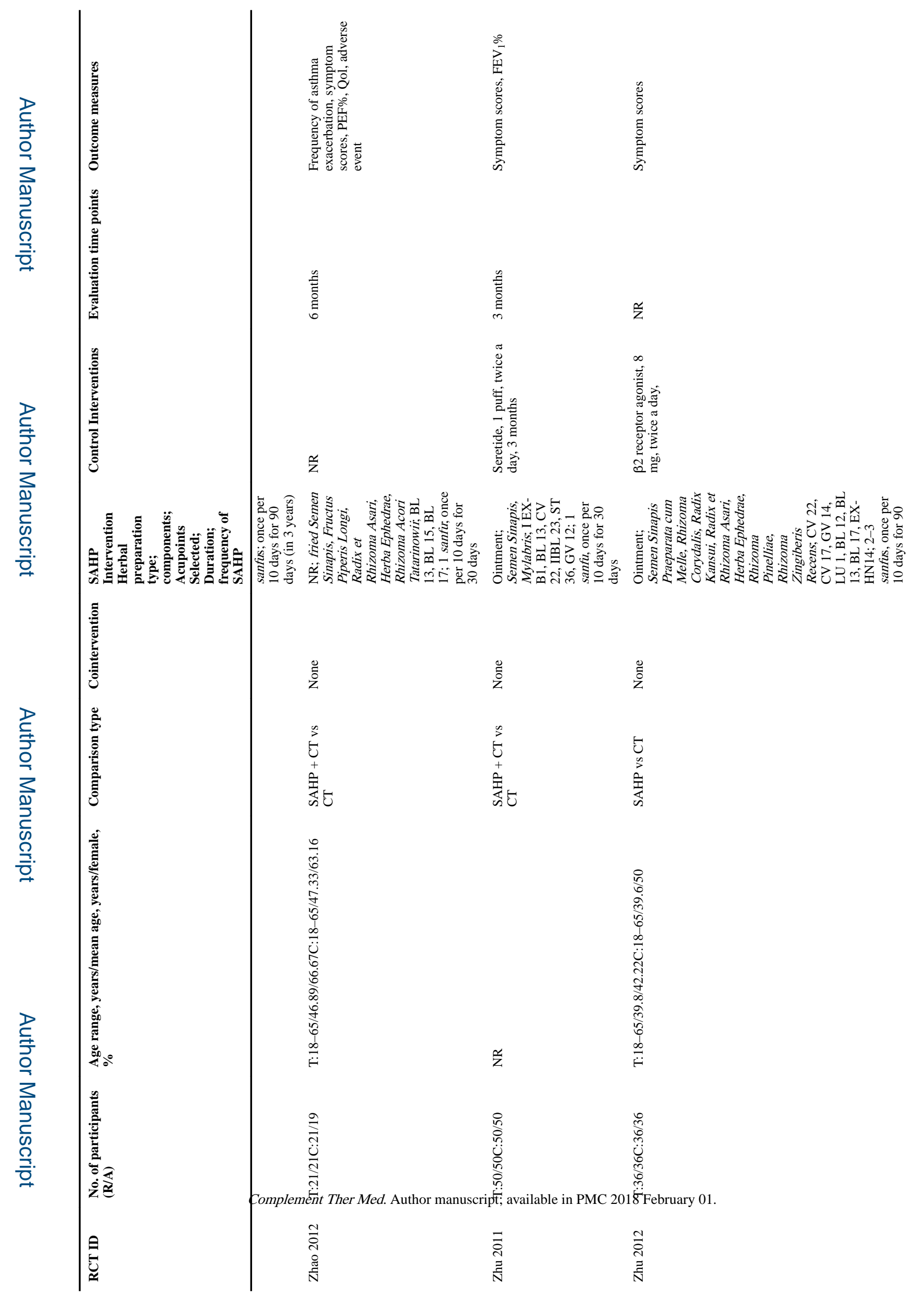




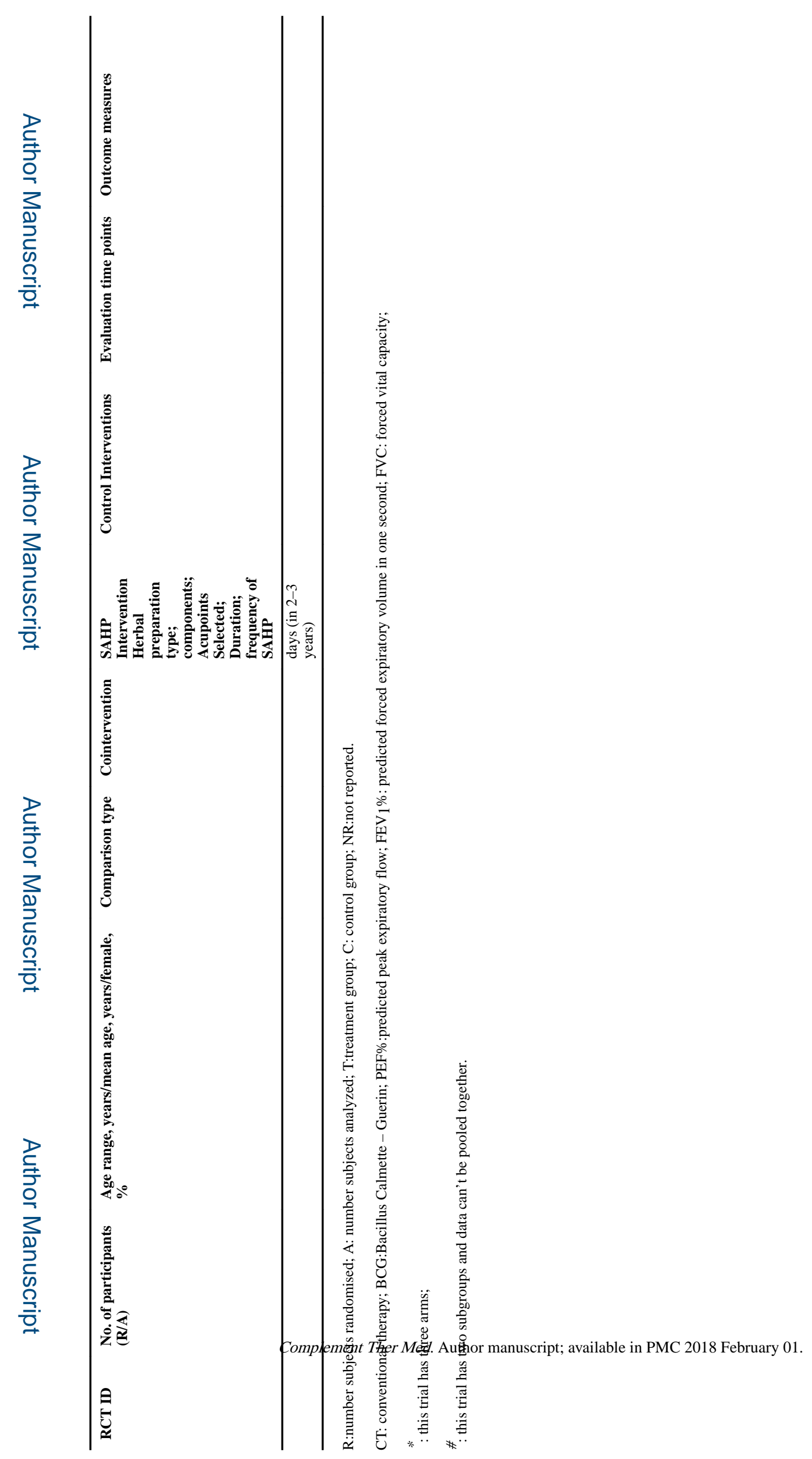




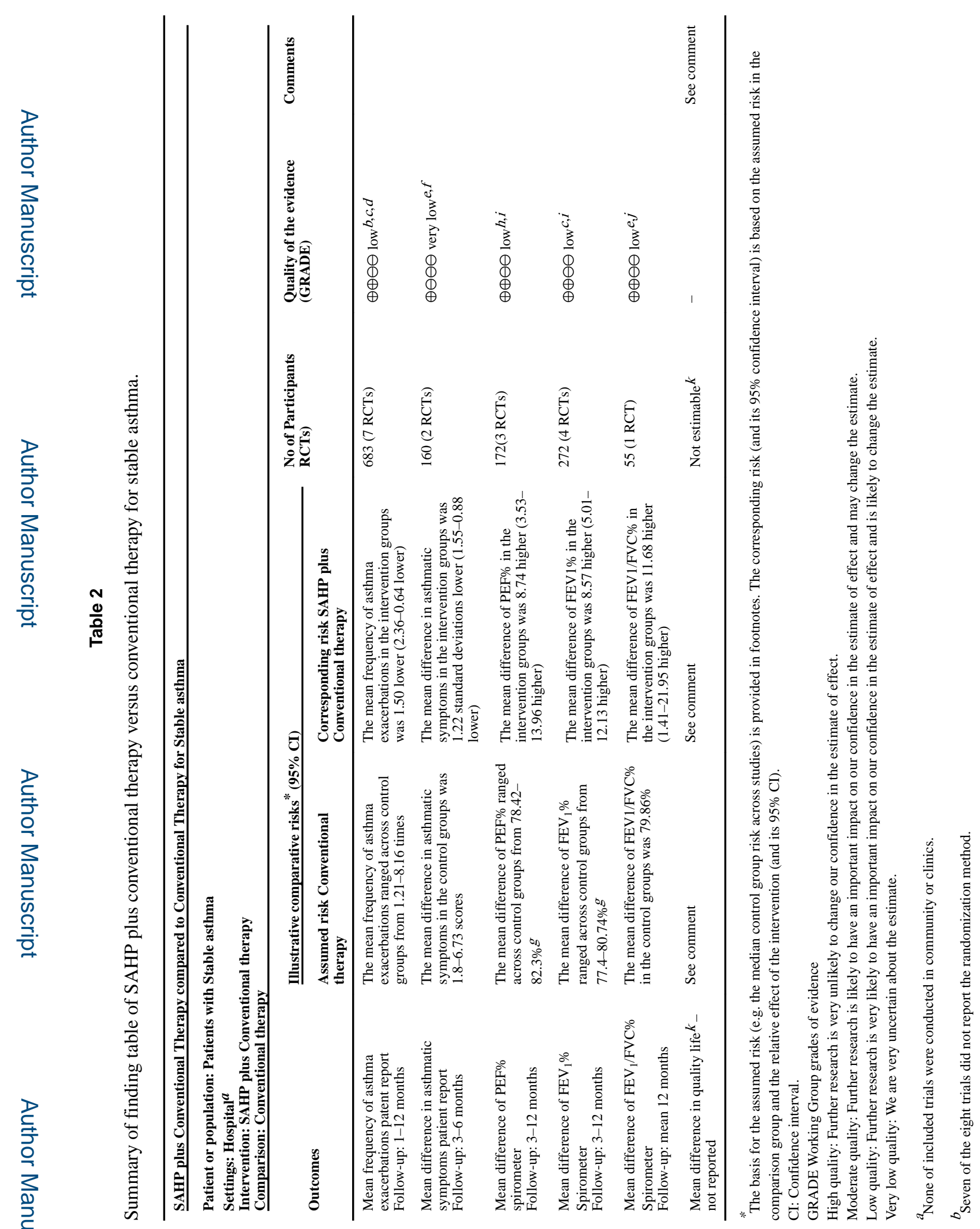

Complement Ther Med. Author manuscript; available in PMC 2018 February 01. 

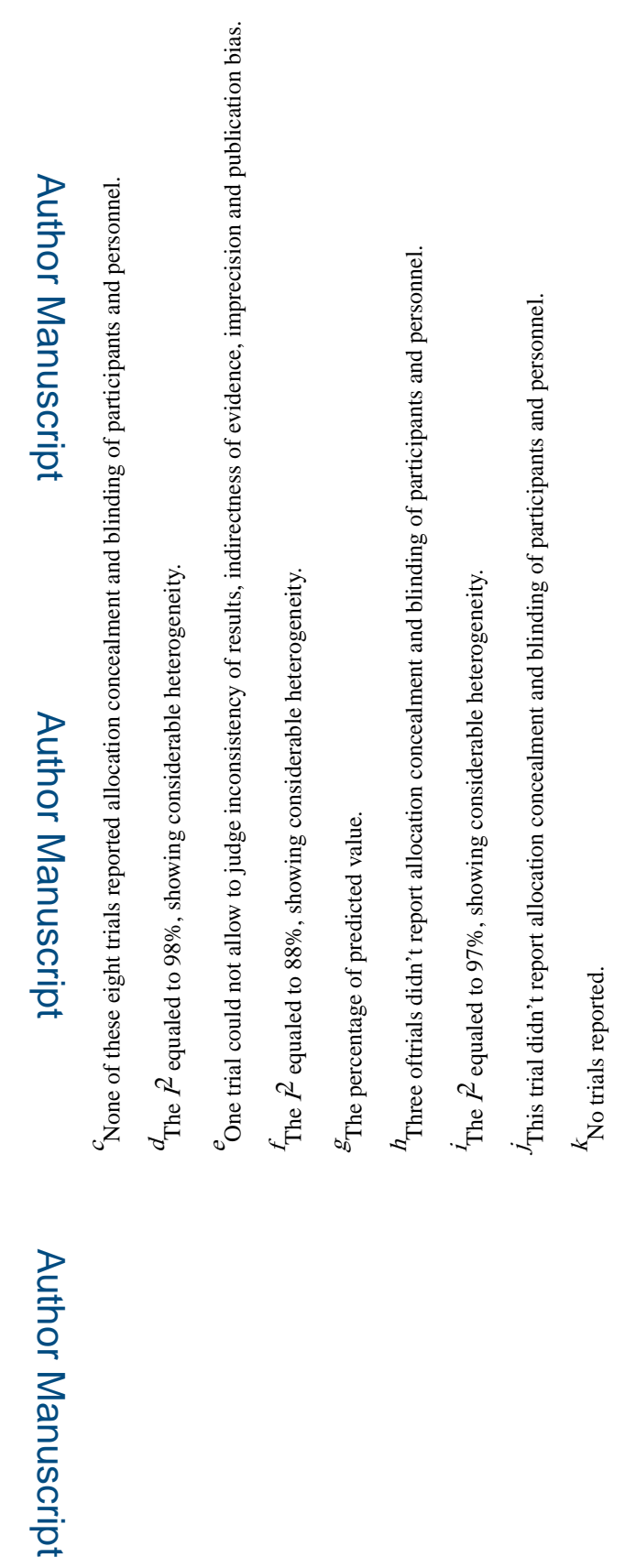

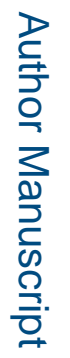

Complement Ther Med. Author manuscript; available in PMC 2018 February 01. 